\title{
New use of current technology to measure rectal temperature and heart rate during endurance exercise
}

\author{
J P Dugas (BSc (Med) (Hons)) ${ }^{1}$ \\ B Burger (BSc (Eng)) $)^{2}$ \\ T D Noakes (MB ChB, PhD, MD) ${ }^{1}$ \\ ${ }^{1}$ MRC/UCT Research Unit for Exercise Science and Sports Medicine, Sports Science Institute of South Africa, University of Cape Town \\ ${ }^{2}$ Sygade Solutions, (Pty) Ltd, Johannesburg
}

\section{Introduction}

The technology necessary to log data remotely and independently has been available for some years. This technology has been applied mostly to environmental and natural sciences, however, and not in life sciences. This was due primarily to the cost of the technology and the small demand for it in the life sciences, especially in studies of exercise physiology. Our recent collaboration with a local technology company (SyGade Solutions (Pty) Ltd., Johannesburg) has resulted in the use of miniature data loggers to record rectal temperature, heart rate (HR), and altitude during road and cycle racing. This technology has the potential to measure these variables simultaneously and in a free-living situation and will therefore contribute to more innovative research.

\section{Description of the data logger}

The data loggers were tested in the laboratory from 35 $44 i \mathrm{C}$, and returned an accuracy ranging from $-0.22 \%$ to $0.1 \%$. Each logger weighs $79 \mathrm{~g}$ and has dimensions of 105 x $58 \times 20 \mathrm{~mm}$ (length $\mathrm{x}$ width $\mathrm{x}$ height, respectively). The loggers use a 16-bit micro-processor operating at $13 \mathrm{Mhz}$, and draw 20 milliamps during operation. Each is powered by a single AAA/1.5 v battery that can supply enough current for up to 12 hours of logging. Data are stored on a FLASH device similar to those used currently in digital cameras. This allows for the retention of data even if power is removed during use.

The loggers can record up to 12 hours of data at 2-second intervals in the absence of HR. When HR is recorded in addition to rectal temperature, the logging time decreases in direct proportion to the time interval between heart beats so that maximum logging time is shortened to 7 hours if a HR of 220 beats/minute is maintained. The minimum HR that can be

\section{CORRESPONDENCE:}

J P Dugas

PO Box 115

Newlands

7725

Tel: $021-6504572$

Fax: 021 - 6867530

E-mail: jdugas@sports.uct.ac.za logged is 30 beats/minute.

The loggers measure HR in milliseconds from beat to beat. Because the HR transmitters are not suited to beat-to-beat calculations, and to reduce interference from other HR monitors, the HR transmitters were hard-wired to the data logger for maximal accuracy.

Temperature is measured by supplying the probe with a very accurate reference voltage/current and then measuring the changes with a 20 bit analog to digital converter. This is then converted to temperature by means of a calibration table, as supplied by the temperature probe manufacturer (VHA Plus, Irving, Texas, USA).

The air pressure sensor is a Motorola MPX4115 which is sensitive to pressure changes within $1 \mathrm{~m}$ of vertical distance. However, for the air pressure sensor to measure altitude accurately in metres above sea level, a sophisticated and accurate calibration is required. Therefore, the loggers were designed not to give accurate meter readings, but instead to give only relative changes in pressure, thus creating a profile of the race course. This allows the user to place an athlete at specific points on a course where changes in altitude are known and frequent.

The software for the micro-processor was compiled initially on a PC and then loaded via a special wire interface to the processor. Data are retrieved by interfacing the logger with a $P C$ via a download cable inserted into the HR transmitter input. The programmer is then able to issue special control commands to the logger via the PC. The data are stored in a raw binary format. The processing and separating of data took place on the PC to produce text files that were then delimited in a Microsoft Excel workbook and plotted using the GraphPad Prism software package (GraphPad Software Inc., San Diego, California, USA).

The loggers contain a function that allows the user to log an event during the data-logging period. The user can log an event by pushing a button on the logger at a point specified by the researcher, for example before, during, or after the race. This allows the researcher to know exactly where the start and finish of the course are, for example, after the data have been downloaded and reduced. 


\section{Pilot work}

Data were logged approximately every 0.03 minutes for the duration of a $100 \mathrm{~km}$ cycle race (Fig. 1) and a $21.1 \mathrm{~km}$ running race (Fig. 2). At the start and finish of each race the athlete logged an event to distinguish the starting and finishing points. The event is marked by a spike in the data set that allows the researcher to identify the event and then remove the spike from the data for presentation purposes. The cycling course profile was then compared with a profile provided by the race organisers to determine specific points on the course during the data session, and these points were then noted on the logger s profile (Fig. 1). The running course had fewer geographical landmarks than the cycling course. Altitude readings from each kilometre on the running course were obtained from the organisers and used to generate a profile of the race course (Fig. 2c). This profile was then compared with the profile created by the logger (Fig. $2 b)$.

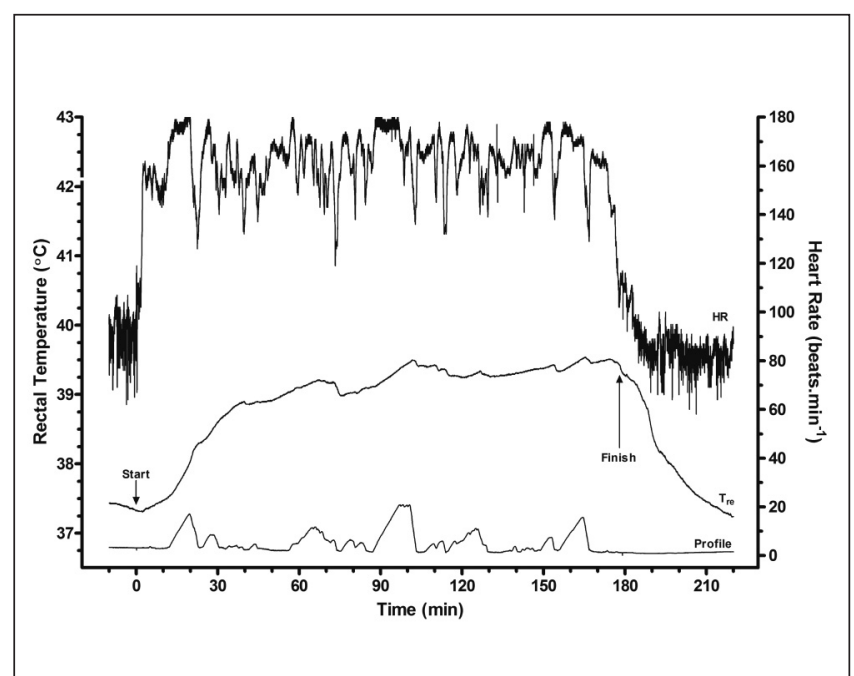

Fig. 1. Data from a $100 \mathrm{~km}$ cycle race. The course profile was measured using an uncalibrated air pressure sensor so that it recorded only relative changes in altitude. The heart rate transmitter was wired directly to the logger for accuracy. Data were logged approximately every 0.03 minutes to produce approximately 60000 data points.

\section{Discussion}

The advent of this technology has already yielded a novel finding. The ability to measure the rectal temperature with such high resolution and over relatively long periods of time has allowed us to show that during endurance exercise rectal temperature is a dynamic variable and appears to change with metabolic rate and course terrain, the former normally being a function of the latter.

In addition, because data of this type have never before been collected, we can see that although the rectal temperature is dynamic in nature as opposed to static, it remains within a range of approximately $2{ }_{\mathrm{i}} \mathrm{C}$. This sheds new light on thermoregulatory studies, and opens new avenues of research in this area that were previously not available.
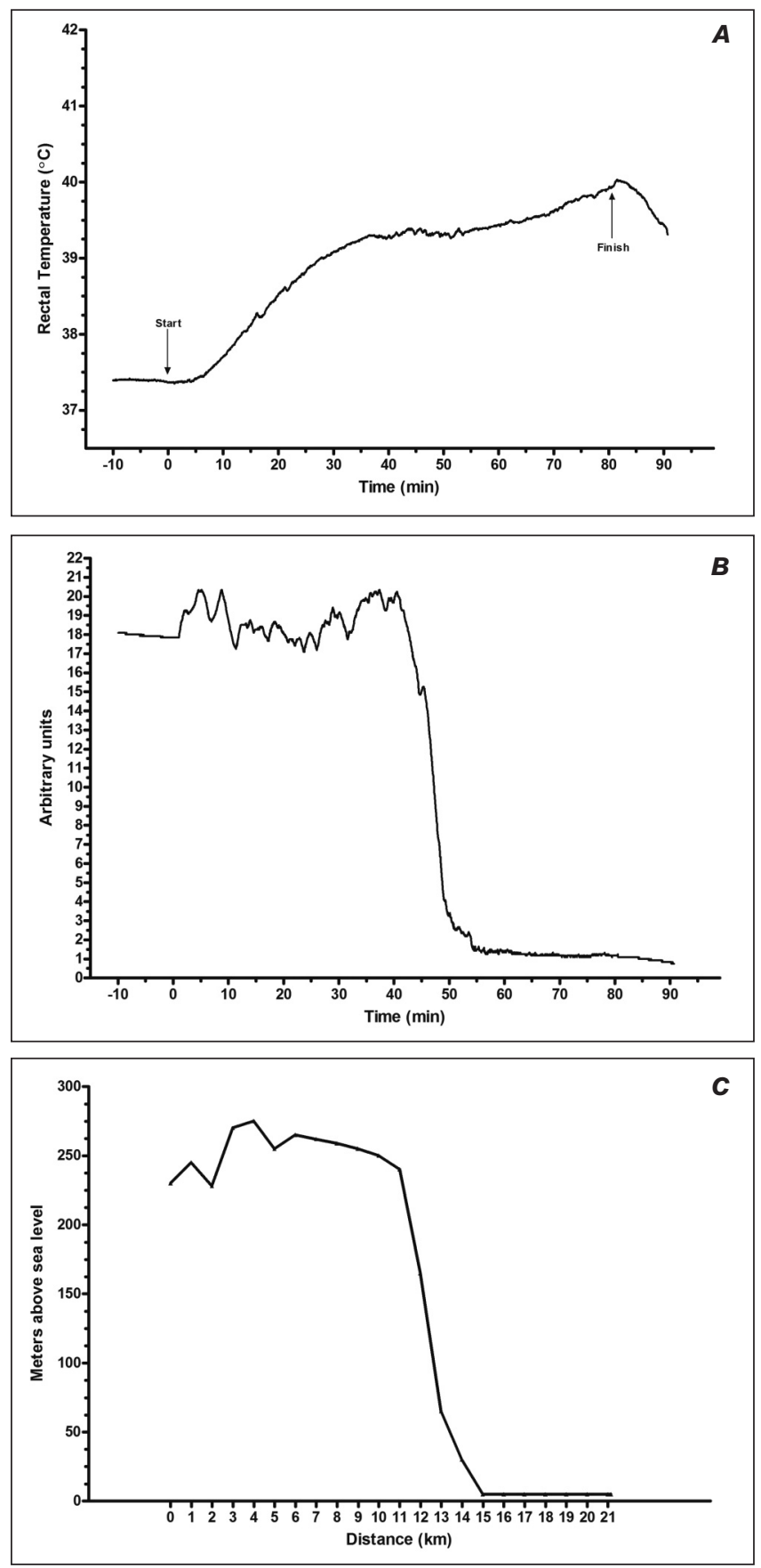

Fig. 2. Data from a $21.1 \mathrm{~km}$ running race. Rectal temperature (2A) and air pressure (2B) were recorded continuously during the run. Altitude at each kilometre (2C) was obtained from the race organisers for comarison with the course profile generated from the logger. 